PROCEEDINGS OF THE

AMERICAN MATHEMATICAL SOCIETY

Volume 140, Number 5, May 2012, Pages 1495-1500

S 0002-9939(2011)11004-9

Article electronically published on August 18, 2011

\title{
SIMPLE RELATIONS IN THE CREMONA GROUP
}

\author{
JÉRÉMY BLANC
}

(Communicated by Ken Ono)

\begin{abstract}
We give a simple set of generators and relations for the Cremona group of the plane. Namely, we show that the Cremona group is the amalgamated product of the de Jonquières group with the group of automorphisms of the plane, divided by one relation which is $\sigma \tau=\tau \sigma$, where $\tau=(x: y: z) \mapsto(y: x: z)$ and $\sigma=(x: y: z) \rightarrow(y z: x z: x y)$.
\end{abstract}

Let $k$ be any fixed algebraically closed field. The Cremona group $\operatorname{Bir}\left(\mathbb{P}^{2}\right)$ is the group of birational transformations of the projective plane $\mathbb{P}^{2}=\mathbb{P}_{k}^{2}$.

The classical Noether-Castelnuovo Theorem says that $\operatorname{Bir}\left(\mathbb{P}^{2}\right)$ is generated by the group $\operatorname{Aut}\left(\mathbb{P}^{2}\right) \cong \operatorname{PGL}(3, k)$, which we will denote by $\mathbf{A}$, and by the standard quadratic transformation

$$
\sigma:(X: Y: Z) \rightarrow(Y Z: X Z: X Y) .
$$

For a proof which is valid over any algebraically closed field (in particular, in any characteristic), see for example [Sha, Chapter V, $\S 5$, Theorem 2, page 100].

A presentation of $\operatorname{Bir}\left(\mathbb{P}^{2}\right)$ was given in $\mathrm{Giz}$. The generators are all the quadratic transformations of the plane (among them, all elements of the form $a_{1} \sigma a_{2}$, where $a_{1}, a_{2} \in \mathbf{A}$ ), and the relations are all those of the form $q_{1} q_{2} q_{3}=1$, where $q_{i}$ is a quadratic map. The proof is quite long and uses many sophisticated tools of algebraic geometry, such as cell complexes associated to rational surfaces.

Another presentation was given in Isk2 (and announced in [Isk1]). The surface taken here is $\mathbb{P}^{1} \times \mathbb{P}^{1}$, and the generators used are the group Aut $\left(\mathbb{P}^{1} \times \mathbb{P}^{1}\right)$ and the de Jonquières group $\mathbf{J}$ of birational maps of $\mathbb{P}^{1} \times \mathbb{P}^{1}$ which preserve the first projection (see below). There is only one relation in the amalgamated product of these two groups, which is $(\rho \tau)^{3}=\sigma$, where $\rho=(x, y) \mapsto(x, x / y)$ and $\tau=(x, y) \mapsto(y, x)$ in local coordinates. The proof is much shorter than the one of [Giz, and the number of relations is also much smaller, but everything is now on $\operatorname{Bir}\left(\mathbb{P}^{1} \times \mathbb{P}^{1}\right)$. There is also some gap in the proof (observed by S. Lamy): the author implicitly uses relations of the form $\left(\rho^{\prime} \tau\right)^{3}=\sigma^{\prime}$ where $\rho^{\prime}$ has base-points infinitely near, without proving that they are generated by the first one (a fact not so hard to prove).

In this short paper, we give a new presentation of the Cremona group, which is as simple as the one of [Isk2, but stays on $\mathbb{P}^{2}$. The proof is also very short and is in fact strongly inspired from the one of Isk2]. We take care of infinitely near points and translate the idea of Iskovskikh from $\mathbb{P}^{1} \times \mathbb{P}^{1}$ to $\mathbb{P}^{2}$, where it becomes

Received by the editors October 29, 2010 and, in revised form, January 11, 2011.

2010 Mathematics Subject Classification. Primary 20F05, 14E07.

This work was supported by SNSF grant No. PP00P2_128422/1.

(C)2011 American Mathematical Society Reverts to public domain 28 years from publication 
simpler. We use only classical tools of plane birational geometry (base-points and blow-ups), as mathematicians of the nineteenth century did and as in [Isk2].

The de Jonquières group, that we will denote by $\mathbf{J}$, is the subgroup of $\operatorname{Bir}\left(\mathbb{P}^{2}\right)$ consisting of elements which preserve the pencil of lines passing through $p_{1}=(1$ : $0: 0)$. This group can be viewed in local coordinates $x=X / Z$ and $y=Y / Z$ as

$$
\mathbf{J}=\left\{\begin{array}{l|l}
(x, y) \rightarrow\left(\frac{a x+b}{c x+d}, \frac{\alpha(x) y+\beta(x)}{\gamma(x) y+\delta(x)}\right) & \begin{array}{l}
\left(\begin{array}{cc}
a & b \\
c & d
\end{array}\right) \in \operatorname{PGL}(2, k), \\
\left(\begin{array}{ll}
\alpha & \beta \\
\gamma & \delta
\end{array}\right) \in \operatorname{PGL}(2, k(x))
\end{array}
\end{array}\right\}
$$

It is thus naturally isomorphic to $\operatorname{PGL}(2, k(x)) \rtimes \operatorname{PGL}(2, k)$, where $\operatorname{PGL}(2, k)=$ $\operatorname{Aut}\left(\mathbb{P}^{1}\right)$ acts on $\operatorname{PGL}(2, k(x))$ via its action on $k(x)=k\left(\mathbb{P}^{1}\right)$.

Since $\sigma \in \mathbf{J}$, the group $\operatorname{Bir}\left(\mathbb{P}^{2}\right)$ is generated by $\mathbf{A}$ and $\mathbf{J}$. The aim of this paper is to prove the following result:

Theorem 1. The Cremona group $\operatorname{Bir}\left(\mathbb{P}^{2}\right)$ is the amalgamated product of $\mathbf{A}=$ $\operatorname{Aut}\left(\mathbb{P}^{2}\right)$ and $\mathbf{J}$ along their intersection, divided by one relation, which is

$$
\sigma \tau=\tau \sigma
$$

where $\tau \in \mathbf{A}$ is given by $\tau=(X: Y: Z) \mapsto(Y: X: Z)$.

Since $\sigma \tau=\tau \sigma$ is easy to verify, it suffices to prove that no other relation holds. We prove this after proving the following simple lemma.

Lemma 1. If $\theta \in \mathbf{J}$ is a quadratic map having $p_{1}=(1: 0: 0)$ and $q$ as base-points, where $q$ is a proper point of $\mathbb{P}^{2} \backslash\left\{p_{1}\right\}$ and $\nu \in \mathbf{A}$ exchanges $p_{1}$ and $q$, the map $\theta^{\prime}=\nu \theta \nu^{-1}$ belongs to $\mathbf{J}$ and the relation

$$
\nu \theta^{-1}=\left(\theta^{\prime}\right)^{-1} \nu
$$

is generated by the relation $\sigma \tau=\tau \sigma$ in the amalgamated product of $\mathbf{A}$ and $\mathbf{J}$.

Proof of Lemma 1. The relations $\theta^{\prime}=\nu \theta \nu^{-1}$ and $\nu \theta^{-1}=\left(\theta^{\prime}\right)^{-1} \nu$ are clearly equivalent. In particular, the result is invariant under conjugation of both $\theta$ and $\nu$ by an element of $\mathbf{A} \cap \mathbf{J}$. Observe that $\mathbf{A} \cap \mathbf{J}$ is the group of automorphisms of $\mathbb{P}^{2}$ which fix the point $p_{1}$. Choosing an element in $\mathbf{A} \cap \mathbf{J}$ which sends $q$ onto $p_{2}=(0: 1: 0)$, we can assume that $q=p_{2}$. Then $\nu$ is equal to $a \tau$, where $\tau=(X: Y: Z) \mapsto(Y: X: Z)$ and $a$ is an element of $\mathbf{A} \cap \mathbf{J}$ which fixes $p_{2}$. We can moreover assume that $\nu=\tau$, since this only replaces $\theta^{\prime}$ with its conjugation by $a \in \mathbf{A} \cap \mathbf{J}$.

We study two cases separately, depending on the number of proper base-points of $\theta$.

(a) Suppose that $\theta$ has exactly three proper base-points, $p_{1}, p_{2}$ and $p_{3}$. Choose $a_{1} \in \mathbf{A} \cap \mathbf{J}$ which fixes $p_{2}$ (and $\left.p_{1}\right)$ and sends $p_{3}$ onto $(0: 0: 1)$ (this is possible since $p_{3}$ is not collinear with $p_{1}$ and $p_{2}$ ). The two maps $\sigma a_{1}$ and $\theta$ are quadratic maps with the same base-points: $p_{1}, p_{2}, p_{3}$. This means that $\theta=a_{2} \sigma a_{1}$ for some $a_{2} \in \mathbf{A}$. Since $\theta, \sigma, a_{1} \in \mathbf{J}$, we see that $a_{1}, a_{2} \in \mathbf{A} \cap \mathbf{J}$. This yields the following equality in the amalgamated product:

$$
\tau \theta \tau^{-1}=\tau a_{2} \sigma a_{1} \tau^{-1}=\left(\tau a_{2} \tau^{-1}\right)\left(\tau \sigma \tau^{-1}\right)\left(\tau a_{1} \tau^{-1}\right) .
$$

Since $\tau, a_{1}, a_{2} \in \mathbf{A}$ and $\tau a_{i} \tau^{-1} \in \mathbf{A} \cap \mathbf{J}$ for $i=1,2$, the above equality implies that $\tau \theta \tau^{-1}$ is equal to an element of $\mathbf{J}$ modulo the relation $\sigma \tau=\tau \sigma$ and therefore yields the result. 
(b) Suppose now that $\theta$ has only two proper base-points, $p_{1}, p_{2}$, and that its third base-point is infinitely near to $p_{i}$ for some $i \in\{1,2\}$. Choose $a_{1} \in \mathbf{A} \cap \mathbf{J}$ which fixes $p_{2}$ (and $p_{1}$ ) and sends this infinitely near point to the tangent direction $z=0$ at the point $p_{i}$. Once again this is possible since the three base-points of $\theta$ are not "collinear".

The maps $\theta$ and $\nu_{i} a_{1}$ are quadratic maps with the same base-points, where $\nu_{1}$, $\nu_{2}$ are the following quadratic involutions:

$$
\begin{array}{lll}
\nu_{1}: & (X: Y: Z) \rightarrow & \left(X Y: Z^{2}: Y Z\right), \\
\nu_{2}: & (X: Y: Z)-\rightarrow & \left(Z^{2}: X Y: X Z\right) .
\end{array}
$$

This means that $\theta=a_{1} \nu_{i} a_{2}$ for some $a_{2} \in \mathbf{A}$. Once again, since $\theta, \sigma, a_{1} \in \mathbf{J}$, we see that $a_{1}, a_{2} \in \mathbf{A} \cap \mathbf{J}$.

Denoting by $\rho_{1}, \rho_{2} \in \mathbf{A} \cap \mathbf{J}$ the maps

$$
\begin{array}{lll}
\rho_{1}: & (X: Y: Z)-\rightarrow & (X: Z-Y: Z), \\
\rho_{2}: \quad & (X: Y: Z)-\rightarrow & (Z-X: Y: Z),
\end{array}
$$

a direct calculation shows that $\nu_{i}=\rho_{i} \sigma \rho_{i} \sigma \rho_{i}$ in $\mathbf{J}$ for $i=1,2$. As above, this yields the following equality:

$$
\tau \theta \tau^{-1}=\left(\tau a_{1} \tau^{-1}\right)\left(\tau \rho_{i} \tau^{-1}\right)\left(\tau \sigma \tau^{-1}\right)\left(\tau \rho_{i} \tau^{-1}\right)\left(\tau \sigma \tau^{-1}\right)\left(\tau \rho_{i} \tau^{-1}\right)\left(\tau a_{2} \tau^{-1}\right)
$$

Using $\sigma \tau=\tau \sigma$ and the fact that $\tau \rho_{i} \tau^{-1}=\rho_{j}$ in $\mathbf{A}$, with $j=3-i$, we obtain

$$
\tau \theta \tau^{-1}=\left(\tau a_{1} \tau^{-1}\right)\left(\rho_{j} \sigma \rho_{j} \sigma \rho_{j}\right)\left(\tau a_{2} \tau^{-1}\right)=\left(\tau a_{1} \tau^{-1}\right) \nu_{j}\left(\tau a_{2} \tau^{-1}\right) .
$$

Therefore, $\tau \theta \tau^{-1}$ is again equal to an element of $\mathbf{J}$ modulo the relation $\sigma \tau=$ $\tau \sigma$.

Proof of Theorem 1. Taking an element $f$ in the amalgamated product $\mathbf{A} \star \mathbf{A} \cap \mathbf{J} \mathbf{J}$ which corresponds to the identity map of $\operatorname{Bir}\left(\mathbb{P}^{2}\right)$, we have to prove that $f$ is the identity in the amalgamated product, modulo the relation $\sigma \tau=\tau \sigma$.

We write $f=j_{r} a_{r} \ldots j_{1} a_{1}$, where $a_{i} \in \mathbf{A}, j_{i} \in \mathbf{J}$ for $i=1, \ldots, r$. Note that many such writings exist, and we allow the $a_{i}, j_{i}$ to be trivial (equal to the identity).

We denote by $\Lambda_{0}$ the linear system of lines of the plane, and for $i=1, \ldots, n$, we denote by $\Lambda_{i}$ the linear system $j_{i} a_{i} \ldots j_{1} a_{1}\left(\Lambda_{0}\right)$ and by $d_{i}$ its degree. We define

$$
D=\max \left\{d_{i} \mid i=1, \ldots, r\right\}, n=\max \left\{i \mid d_{i}=D\right\} \text { and } k=\sum_{i=1}^{n}\left(\operatorname{deg}\left(j_{i}\right)-1\right) .
$$

Recall that each $j_{i}$ is a birational transformation of the plane and that it has a degree $\operatorname{deg}\left(j_{i}\right)$, which is equal to the degree of the linear systems $j_{i}\left(\Lambda_{0}\right)$ and $\left(j_{i}\right)^{-1}\left(\Lambda_{0}\right)$. In particular, $\operatorname{deg}\left(j_{i}\right)=1 \Leftrightarrow j_{i} \in \mathbf{A}$. The number $k$ counts the complexity of the word $j_{n} a_{n} \ldots j_{1} a_{1}$, which corresponds to the birational map $j_{i} a_{i} \ldots j_{1} a_{1}$ of the highest degree.

When $D=1$, each $j_{i}$ belongs to $\mathbf{A}$, and the word $f$ is equal to an element of $\mathbf{A}$ in the amalgamated product. Since $\mathbf{A}$ embeds into $\operatorname{Bir}\left(\mathbb{P}^{2}\right)$, this case is clear. We can thus assume that $D>1$ and prove the result by induction on the pairs $(D, k)$, ordered lexicographically.

If $j_{n}$ belongs to $\mathbf{A}$, we replace $a_{n+1} j_{n} a_{n}$ by its product in $\mathbf{A}$. This does not change the pair $(D, k)$ but decreases $n$ by 1 . If $j_{n+1}$ belongs to $\mathbf{A}$, we replace $a_{n+2} j_{n+1} a_{n+1}$ by its product in $\mathbf{A}$ and decrease $r$ by 1 without changing the pair $(D, k)$. If $a_{n+1} \in \mathbf{J}$ we replace $j_{n+1} a_{n+1} j_{n}$ by its product in $\mathbf{J}$, decreasing the pair $(D, k)$. 
We can thus assume that $j_{n+1}, j_{n} \in \mathbf{J} \backslash \mathbf{A}$ and that $a_{n+1} \in \mathbf{A} \backslash \mathbf{J}$. Remembering that $p_{1}=(1: 0: 0)$ is the base-point of the pencil associated to $\mathbf{J}$, the fact that $a_{n+1} \notin \mathbf{J}$ implies that $a_{n+1}\left(p_{1}\right) \neq p_{1}$. Moreover, the fact that $j_{n}, j_{n+1} \notin \mathbf{A}$ implies that both have degree $>1$. We will denote these degrees by $D_{L}=\operatorname{deg}\left(j_{n+1}\right)$, $D_{R}=\operatorname{deg}\left(j_{n}\right)$. Since $j_{n+1}$ and $j_{n}$ preserve the pencil of lines passing through $p_{1}$, the point $p_{1}$ is a base-point of $j_{n+1}$ and $j_{n}$ of multiplicity $D_{L}-1$ and $D_{R}-1$ respectively. This means that the systems $\left(j_{n+1}\right)^{ \pm 1}\left(\Lambda_{0}\right)$ and $\left(j_{n}\right)^{ \pm 1}\left(\Lambda_{0}\right)$ consist of curves of degree $D_{L}$ and $D_{R}$ having multiplicity $D_{L}-1$ and $D_{R}-1$ at $p_{1}$. These systems, being the image of the system $\Lambda_{0}$ of lines of the plane, are homoloidal nets (systems of rational curves with exactly one free intersection point). They have thus respectively $2 D_{L}-2$ and $2 D_{R}-2$ base-points distinct from $p_{1}$, which all have multiplicity one.

We denote by $\Omega_{L}$ and $\Omega_{R}$ the systems

$$
\Omega_{L}=\left(j_{n+1} a_{n+1}\right)^{-1}\left(\Lambda_{0}\right) \text { and } \Omega_{R}=j_{n} a_{n}\left(\Lambda_{0}\right)
$$

which have degree $D_{L}$ and $D_{R}$ respectively, since $a_{n}, a_{n+1} \in \mathbf{A}=\operatorname{Aut}\left(\mathbb{P}^{2}\right)$ are only changes of coordinates. Moreover, the points $l_{0}=\left(a_{n+1}\right)^{-1}\left(p_{1}\right) \neq p_{1}$ and $r_{0}=p_{1}$ are base-points of respectively $\Omega_{L}$ and $\Omega_{R}$ of multiplicity $D_{L}-1$ and $D_{R}-1$.

We use these systems to compute the degrees $d_{n+1}$ and $d_{n-1}$ of the systems $\Lambda_{n+1}=j_{n+1} a_{n+1}\left(\Lambda_{n}\right)$ and $\Lambda_{n-1}=\left(a_{n}\right)^{-1}\left(j_{n}\right)^{-1}\left(\Lambda_{n}\right)$. For any $i, d_{i}$ is equal to the degree of $\Lambda_{i}$, which is the intersection of $\Lambda_{i}$ with a general line, or the free intersection of $\Lambda_{i}$ with $\Lambda_{0}$. In particular, $d_{n+1}$ is equal to the free intersection of $\Lambda_{n+1}=\left(j_{n+1} a_{n+1}\right)\left(\Lambda_{n}\right)$ with $\Lambda_{0}$ or to the free intersection of $\Lambda_{n}$ with $\Omega_{L}$. Similarly, $d_{n-1}$ is equal to the free intersection of $\Lambda_{n+1}=\left(j_{n+1} a_{n+1}\right)\left(\Lambda_{n}\right)$ with $\Lambda_{0}$ or to the free intersection of $\Lambda_{n}$ with $\Omega_{L}$.

Writing $l_{1}, \ldots, l_{2 D_{L}-2}$ and $r_{1}, \ldots, r_{2 D_{R}-2}$ as the other base-points of $\Omega_{L}$ and $\Omega_{R}$ (which have all multiplicity one, as we said above), we obtain the following equalities:

$$
\begin{aligned}
& d_{n+1}=D_{L} \cdot d_{n}-\left(D_{L}-1\right) \cdot m\left(l_{0}\right)-\sum_{i}^{2 D_{L}-2} m\left(l_{i}\right)<d_{n}, \\
& d_{n-1}=D_{R} \cdot d_{n}-\left(D_{R}-1\right) \cdot m\left(r_{0}\right)-\sum_{i}^{2 D_{R}-2} m\left(r_{i}\right) \leq d_{n},
\end{aligned}
$$

where $m(q) \geq 0$ is the multiplicity of a point $q$ as a base-point of $\Lambda_{n}$. We order the points $l_{1}, \ldots, l_{2 D_{L}-2}$ so that $m\left(l_{i}\right) \geq m\left(l_{i+1}\right)$ for each $i \geq 1$ and that if $l_{i}$ is infinitely near to $l_{j}$, then $i>j$. We do the same for $r_{1}, \ldots, r_{2 D_{R}-2}$. Rewriting the above inequalities as

$$
\begin{aligned}
d_{n+1} & =d_{n}+\sum_{i=1}^{D_{L}-1}\left(d_{n}-m\left(l_{0}\right)-m\left(l_{2 i-1}\right)-m\left(l_{2 i}\right)\right)<d_{n}, \\
d_{n-1} & =d_{n}+\sum_{i=1}^{D_{R}-1}\left(d_{n}-m\left(r_{0}\right)-m\left(r_{2 i-1}\right)-m\left(r_{2 i}\right)\right) \leq d_{n},
\end{aligned}
$$

the order chosen implies that

$$
\begin{aligned}
m\left(l_{0}\right)+m\left(l_{1}\right)+m\left(l_{2}\right) & >d_{n}, \\
m\left(r_{0}\right)+m\left(r_{1}\right)+m\left(r_{2}\right) & \geq d_{n} .
\end{aligned}
$$

(a) Suppose that $m\left(l_{0}\right) \geq m\left(l_{1}\right)$ and $m\left(r_{0}\right) \geq m\left(r_{1}\right)$. We choose a point $q$ in the set $\left\{l_{1}, l_{2}, r_{1}, r_{2}\right\} \backslash\left\{l_{0}, r_{0}\right\}$ with the maximal multiplicity $m(q)$, and so that $q$ is a proper point of the plane or infinitely near to $l_{0}$ or $r_{0}$ (which are distinct proper points of the plane). We now prove that

$$
m\left(l_{0}\right)+m\left(r_{0}\right)+m(q)>d_{n} .
$$


If $l_{1}=r_{0}, m(q) \geq m\left(l_{2}\right)$ and $m\left(l_{0}\right)+m\left(r_{0}\right)+m(q) \geq m\left(l_{0}\right)+m\left(l_{1}\right)+m\left(l_{2}\right)>d_{n}$ by (11). If $l_{1} \neq r_{0}, m(q) \geq m\left(l_{1}\right) \geq m\left(l_{2}\right)$ so $m\left(l_{0}\right)+m(q)>2 d_{n} / 3$. Since $m\left(r_{0}\right) \geq m\left(r_{1}\right) \geq m\left(r_{2}\right)$, we have $m\left(r_{0}\right) \geq d_{n} / 3$, and inequality (2) is clear.

Because of inequality (2), the points $l_{0}, r_{0}$ and $q$ are not aligned, and there exists a quadratic map $\theta \in \mathbf{J}$ with base-points $l_{0}, r_{0}, q$ (recall that $r_{0}=p_{1}$ is the point associated to the pencil of $\mathbf{J})$. Moreover, the degree of $\theta\left(\Lambda_{n}\right)$ is $2 d_{n}-m\left(l_{0}\right)-m\left(r_{0}\right)-$ $m(q)<d_{n}$. Recall that $a_{n+1} \in \mathbf{A}$ sends $l_{0}$ onto $r_{0}=p_{1}$. Choosing $\nu \in \mathbf{A} \cap \mathbf{J}$ which (fixes $r_{0}=p_{1}$ and) sends $a_{n+1}\left(r_{0}\right)$ onto $l_{0}$ and replacing respectively $a_{n+1}$ and $j_{n+1}$ by $\nu a_{n+1}$ and $j_{n+1} \nu^{-1}$, we can assume that $a_{n+1}$ exchanges $l_{0}$ and $r_{0}$. Using Lemma 1, we write $\theta^{\prime}=a_{n+1} \theta\left(a_{n+1}\right)^{-1} \in \mathbf{J}$ and obtain the following equality modulo the relation $\sigma \tau=\tau \sigma$ :

$$
j_{n+1} a_{n+1} j_{n}=j_{n+1} a_{n+1} \theta^{-1}\left(\theta j_{n}\right)=\left(j_{n+1}\left(\theta^{\prime}\right)^{-1}\right) a_{n+1}\left(\theta j_{n}\right),
$$

and both $\left(j_{n+1}\left(\theta^{\prime}\right)^{-1}\right)$ and $\left(\theta j_{n}\right)$ belong to $\mathbf{J}$, but $a_{n+1} \in \mathbf{A}$. Since $\theta\left(\Lambda_{n}\right)=$ $\left(\theta j_{n}\right)\left(\Lambda_{n-1}\right)$ has degree $<d_{n}$, this rewriting decreases the pair $(D, k)$.

(b) Suppose now that we are in a "bad case" where $m\left(l_{0}\right)<m\left(l_{1}\right)$ or $m\left(r_{0}\right)<$ $m\left(r_{1}\right)$. We now prove that it is possible to change the writing of $f$ in the amalgamated product (modulo the relation) without changing $(D, k)$ but by reversing the inequalities. We will thus be able to go back to the "good case" already studied in (a) to conclude.

Assume first that $m\left(r_{1}\right)>m\left(r_{0}\right)$. This implies that $r_{1}$ is a proper point of the plane and that there exists a quadratic map $\theta \in \mathbf{J}$ with base-points $p_{1}=r_{0}, r_{1}, r_{2}$. Since these three points are base-points of $\left(j_{n}\right)^{-1}$, the degree of $\theta j_{n} \in \mathbf{J}$ is equal to the degree of $j_{n} \in \mathbf{J}$ minus 1 .

Taking $\nu \in \mathbf{A}$ which exchanges $r_{0}$ and $r_{1}$ and applying Lemma 1 we write $\theta^{\prime}=\nu \theta \nu^{-1} \in \mathbf{J}$ and obtain the following equality modulo the relation $\sigma \tau=\tau \sigma$ :

$$
a_{n+1} j_{n}=\left(a_{n+1} \nu^{-1}\right) \nu \theta^{-1}\left(\theta j_{n}\right)=\left(a_{n+1} \nu^{-1}\right)\left(\theta^{\prime}\right)^{-1} \nu\left(\theta j_{n}\right),
$$

and both $\theta^{\prime}$ and $\left(\theta j_{n}\right)$ belong to $\mathbf{J}$, but $\left(a_{n+1} \nu^{-1}\right)$ and $\nu$ belong to $\mathbf{A}$. Since $\left(\theta^{\prime}\right)^{-1} \nu \theta\left(\Lambda_{n}\right)=\nu\left(\Lambda_{n}\right)$, this rewriting replaces

$$
\begin{aligned}
\left(j_{1}, \ldots, j_{n}, \ldots, j_{r}\right) & \text { with } \quad\left(j_{1}, \ldots, j_{n-1}, \theta j_{n},\left(\theta^{\prime}\right)^{-1}, j_{n+1}, \ldots, j_{r}\right), \\
\left(\Lambda_{0}, \ldots, \Lambda_{n}, \ldots, \Lambda_{r}\right) & \text { with } \quad\left(\Lambda_{0}, \ldots, \Lambda_{n-1}, \theta\left(\Lambda_{n}\right), \nu\left(\Lambda_{n}\right), \Lambda_{n+1}, \ldots, \Lambda_{r}\right) .
\end{aligned}
$$

The degree of $\theta\left(\Lambda_{n}\right)$ is equal to $2 d_{n}-m\left(r_{0}\right)-m\left(r_{1}\right)-m\left(r_{2}\right) \leq d_{n}$, and the degree of $\nu\left(\Lambda_{n}\right)$ is $d_{n}$. The new sequence thus has the same $D, n$ is replaced with $n+1$, and $k$ stays the same since $\operatorname{deg}\left(\left(\theta^{\prime}\right)^{-1}\right)-1+\operatorname{deg}\left(\theta j_{n}\right)-1=2-1+\operatorname{deg}\left(\theta j_{n}\right)-1=\operatorname{deg}\left(j_{n}\right)-1$. The system $\Lambda_{n}$ being replaced with $\nu\left(\Lambda_{n}\right)$, where $\nu \in \mathbf{A}$ exchanges $r_{0}$ and $r_{1}$, the multiplicity of $r_{0}$ as a base-point of $\nu\left(\Lambda_{n}\right)$ is now the biggest among the base-points of $\theta^{\prime}$. In the new sequence, we have $m\left(r_{0}\right)>m\left(r_{1}\right)$ instead of $m\left(r_{1}\right)>m\left(r_{0}\right)$.

If $m\left(l_{1}\right)>m\left(l_{0}\right)$, the same kind of replacement exchanges the points $l_{0}$ and $l_{1}$.

We can thus go back to case $(a)$ after having made one or two replacements. This achieves the proof.

\section{ACKNOWLEDGEMENT}

The authors would like to thank the referee for corrections and precious remarks which helped to provide a gentler exposition. 


\section{REFERENCES}

[Giz] M. Kh. Gizatullin, Defining relations for the Cremona group of the plane, Izv. Akad. Nauk SSSR Ser. Mat. 46 (1982), 909-970; English transl. in Math. USSR Izv. 21 (1983). MR675525 (84e:14010)

[Isk1] V. A. Iskovskikh, Generators and relations in a two-dimensional Cremona group, Vestnik Moskov. Univ. Ser. I Mat. Mekh. 1983, no. 5, 43-48. English transl. in Moscow Univ. Math. Bull. 38 (1983), no. 5, 56-63. MR722450 (85e:14019)

[Isk2] V. A. Iskovskikh, Proof of a theorem on relations in the two-dimensional Cremona group, Uspekhi Mat. Nauk 40 (1985), no. 5 (245), 255-256. English transl. in Russian Math. Surveys 40 (1985), no. 5, 231-232. MR810819 (87d:14007)

[Sha] I.R. Shafarevich, Algebraic surfaces, Proc. Steklov Inst. Math. 75 (1967).

Mathematisches Institut, University of Basel, Rheinsprung 21, CH-4051 Basel, SwitZERLAND

E-mail address: Jeremy.Blanc@unibas.ch 